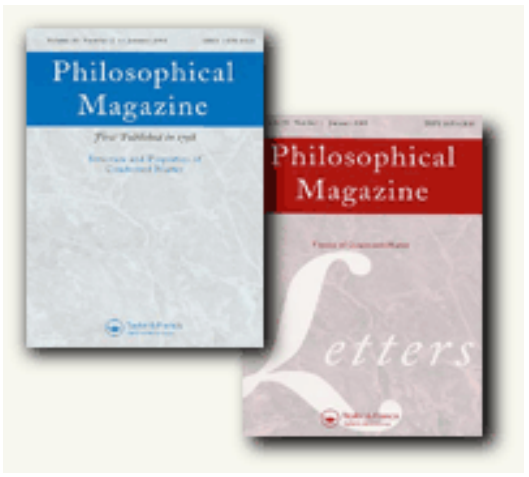

\title{
Structural properties and magnetic interactions in martensitic Ni-Mn-Sb alloys
}

\begin{tabular}{|r|l|}
\hline Journal: & Philosophical Magazine \& Philosophical Magazine Letters \\
\hline Manuscript ID: & TPHM-09-Jan-0037 \\
\hline Journal Selection: & Philosophical Magazine \\
\hline Dathor: & 25-Jan-2009 \\
\hline Complete List of Authors: & $\begin{array}{l}\text { Aksoy, Seda; Duisburg-Essen University, Experimental physics } \\
\text { Acet, Mehmet; Duisburg-Essen University, Experimental physics } \\
\text { Wassermann, Eberhard; Duisburg-Essen University, Experimental } \\
\text { physics } \\
\text { Krenke, Thorsten; ThyssenKrupp Electrical Steel, Forschung \& } \\
\text { Entwicklung } \\
\text { Moya, Xavier; Universitat de Barcelona, Estructura i Constituents de } \\
\text { la Matèria } \\
\text { Manosa, Lluis; Universitat de Barcelona, Estructura i Constituents } \\
\text { de la Matèria } \\
\text { Planes, Antoni; Universitat de Barcelona, Estructura i Constituents } \\
\text { de la Matèria } \\
\text { Deen, Pascale; Institut Laue-Langevin }\end{array}$ \\
\hline Keywords: & $\begin{array}{l}\text { magnetic properties, neutron diffraction, phase diagrams, phase } \\
\text { transitions, polarization }\end{array}$ \\
\hline Keywords (user supplied): & \begin{tabular}{l} 
magnetic properties, neutron diffraction, phase diagrams \\
\hline
\end{tabular} \\
\hline \hline
\end{tabular}

\section{(s) ScholarONE Manuscript Central}


Philosophical Magazine

Vol. 00, No. 00, 25 January 2009, 1-14

\title{
RESEARCH ARTICLE
}

\section{Structural properties and magnetic interactions in martensitic Ni-Mn-Sb alloys}

\author{
Seda Aksoy ${ }^{\mathrm{a}}$, Mehmet Acet ${ }^{\mathrm{a} *}$, Eberhard F. Wassermann ${ }^{\mathrm{a}}$, Thorsten Krenke ${ }^{\mathrm{b}}$, Xavier \\ Moya ${ }^{c}$, Lluís Mañosa ${ }^{c}$, Antoni Planes ${ }^{\mathrm{c}}$ and Pascal P. Deen ${ }^{\mathrm{d}}$ \\ ${ }^{a}$ Experimentalphysik (AG-Farle), Universität Duisburg-Essen, D-47048, Duisburg, \\ Germany; ${ }^{\mathrm{b}}$ ThyssenKrupp Electrical Steel, F\&E Ge, Kurt-Schumacher-Str. 95, D-45881 \\ Gelsenkirchen, Germany; ${ }^{\mathrm{c}}$ Facultat de Física, Departament dEstructura i Constituents de \\ la Matèria, Universitat de Barcelona, Diagonal 647, E-08028 Barcelona, Catalonia, \\ Spain; ${ }^{\mathrm{d}}$ Institut Laue-Langevin, BP 156, 38042 Grenoble Cedex 9, France
}

(Received 00 Month 200x; final version received 00 Month 200x)

\begin{abstract}
We study the structural and magnetic properties of Heusler based $\mathrm{Ni}_{50} \mathrm{Mn}_{50-x} \mathrm{Sb}_{x}$ in the composition range $5.0 \leq x \leq 25.0$. The cubic phase is preserved in the range $17.0 \leq x \leq 25.0$, and the presence of martensitic transformations are found in alloys with $x \leq 15.0$. In a critical composition range $12.5 \leq x \leq 10.0$, the magnetic coupling in both austenitic and martensitic phases is paramagnetic, however the presence of antiferromagnetic short-range correlations is observed in both phases. We discuss the consequences of these correlations on the behavior of the magnetic moment with respect to electronic properties.
\end{abstract}

Keywords: Heusler alloys; magnetisation; neutron polarisation analysis; magnetic correlations; phase diagrams

\section{Introduction}

Ni-Mn-Z-based ( $Z$ : Ga, In, Sn, Sb) ferromagnetic Heusler alloys which transform martensitically from a high-symmetry cubic austenite state to a low-symmetry martensite state exhibit strong coupling between magnetic and structural degrees of freedom leading to the magnetic shape-memory effect particularly in Ni-MnGa alloys [1-3]. Although significant, these effects are not the only ones featured, and it was found that effects such as large magnetic-field-induced strain $[4,5]$, magneto-resistance $[6,7]$, magnetic super-elasticity $[8,9]$ and conventional and inverse magneto-caloric effects $[10,11]$ can also occur in a wider class of $\mathrm{Ni}-\mathrm{Mn}-Z$ Heusler alloys. More recently, the observations of the effect of an external field on nucleation properties of martensite [12] and the kinetic arrest effect [13, 14] have enriched the variety of observed features. To understand the properties leading to these diverse effects featured by Ni-Mn- $Z$ alloys and how these properties are related to one another, it is important to understand the details of the magnetic and structural properties of these systems both in the temperature-vicinity of the martensitic transformation and in temperature ranges well within the martensite and austenite states.

${ }^{*}$ Corresponding author. Email: mehmet.acet@uni-due.de

ISSN: 1478-6435 print/ISSN 1478-6443 online

(C) 2009 Taylor \& Francis

DOI: $10.1080 / 1478643$ YYxxxxxxxx

http://www.informaworld.com 
Table 1. Compositions of the $\mathrm{Ni}_{50} \mathrm{Mn}_{50-x} \mathrm{Sb}_{x}$ samples determined by EDX analysis and the valence electron concentrations $e / a$.

\begin{tabular}{lcccc}
\hline$x$ (nominal) & $\mathrm{Ni}$ & $\mathrm{Mn}$ & $\mathrm{Sb}$ & $e / a$ \\
\hline 25.0 & 50.1 & 24.5 & 25.4 & 7.995 \\
20.0 & 50.0 & 30.1 & 19.9 & 8.102 \\
17.0 & 50.3 & 32.9 & 16.8 & 8.173 \\
15.0 & 49.7 & 35.0 & 15.3 & 8.185 \\
14.0 & 50.3 & 35.9 & 13.8 & 8.233 \\
13.0 & 50.2 & 36.6 & 13.2 & 8.242 \\
12.5 & 51.5 & 36.0 & 12.5 & 8.295 \\
10.0 & 50.3 & 39.6 & 10.1 & 8.307 \\
5.0 & 51.9 & 42.7 & 5.4 & 8.449 \\
\hline
\end{tabular}

In the compositional series $\mathrm{Ni}_{50} \mathrm{Mn}_{50-x} Z_{x}$ the rate of change of the martensitic transformation temperature with respect to composition is fastest when $Z$ is $\mathrm{Sb}$ [15]. $\mathrm{Ni}_{50} \mathrm{Mn}_{50-x} \mathrm{Sb}_{x}$ is also a system where the magnetic moment $\mu$ drops continuously with increasing valence-electron concentration $e / a$, whereas in Heusler and half-Heusler alloys the behavior is generally the opposite. Only at some offstoichiometric compositions of alloys with $Z$ as Ga or Sn can one find behavior where $\mu$ drops with increasing $e / a$. These features make it interesting to look closer to the structural and magnetic properties of $\mathrm{Ni}_{50} \mathrm{Mn}_{50-x} \mathrm{Sb}_{x}$.

Here, we examine further the magnetic and structural properties of $\mathrm{Ni}-\mathrm{Mn}-\mathrm{Sb}$ Heusler alloys by differential scanning calorimetry (DSC), magnetisation, X-ray diffraction, and diffuse neutron scattering methods and use the results together with those obtained in previous studies on Ni-Mn- $Z$ Heusler alloys to provide an understanding of the common features. We study the $e / a$ dependence of $\mu$ and discuss its relation to the observed antiferromagnetic coupling in the austenite and martensite states.

\section{Experimental}

Polycrystalline alloys of Ni-Mn-Sb were prepared by arc melting pure metals under argon atmosphere in a water-cooled $\mathrm{Cu}$ crucible and were remelted several times. The ingots were encapsulated and homogenised under argon in quartz glass and annealed at $1073 \mathrm{~K}$ for 2 hours followed by quenching in ice-water. The compositions of the alloys were determined by energy dispersive $\mathrm{x}$-ray photoluminescence analysis (EDX) and are given in Tab. I with their corresponding $e / a$.

Differential scanning calorimetry (DSC) measurements were carried out in the temperature range $200 \leq T \leq 830 \mathrm{~K}$ (TA Instruments MDSC 2920). The cooling and heating rates were $2-5 \mathrm{Kmin}^{-1}$. One side of the samples were ground with 1200 grid $\mathrm{SiC}$ abrasive to insure proper thermal contact.

X-ray diffraction (XRD) on powder samples at room temperature were carried out on an X'Pert Pro Diffractometer using Co $K \alpha$ radiation. Neutron polarisation analysis experiments were carried out for the alloy with $x=10.0$ in the martensite and austenite states on the D7 diffuse scattering spectrometer at the Institut LaueLangevin, Grenoble using neutrons of wavelength 4.86 A. The D7 instrument allows full XYZ polarisation analysis to separate nuclear and magnetic cross-sections $(d \sigma / d \Omega)_{N U C}$ and $(d \sigma / d \Omega)_{M A G}[16]$.

The temperature dependence of the magnetisation $M(T)$ under $H=5 \mathrm{mT}$ and $H=5 \mathrm{~T}$ were measured in the temperature range $5 \mathrm{~K} \leq T \leq 380 \mathrm{~K}$ using a superconducting quantum interference device magnetometer. Prior to the measurements, the sample was prepared in a zero-field-cooled state (ZFC) by cooling it from 380 $\mathrm{K}$ to $5 \mathrm{~K}$ in the absence of a magnetic field and then the external field was ap- 


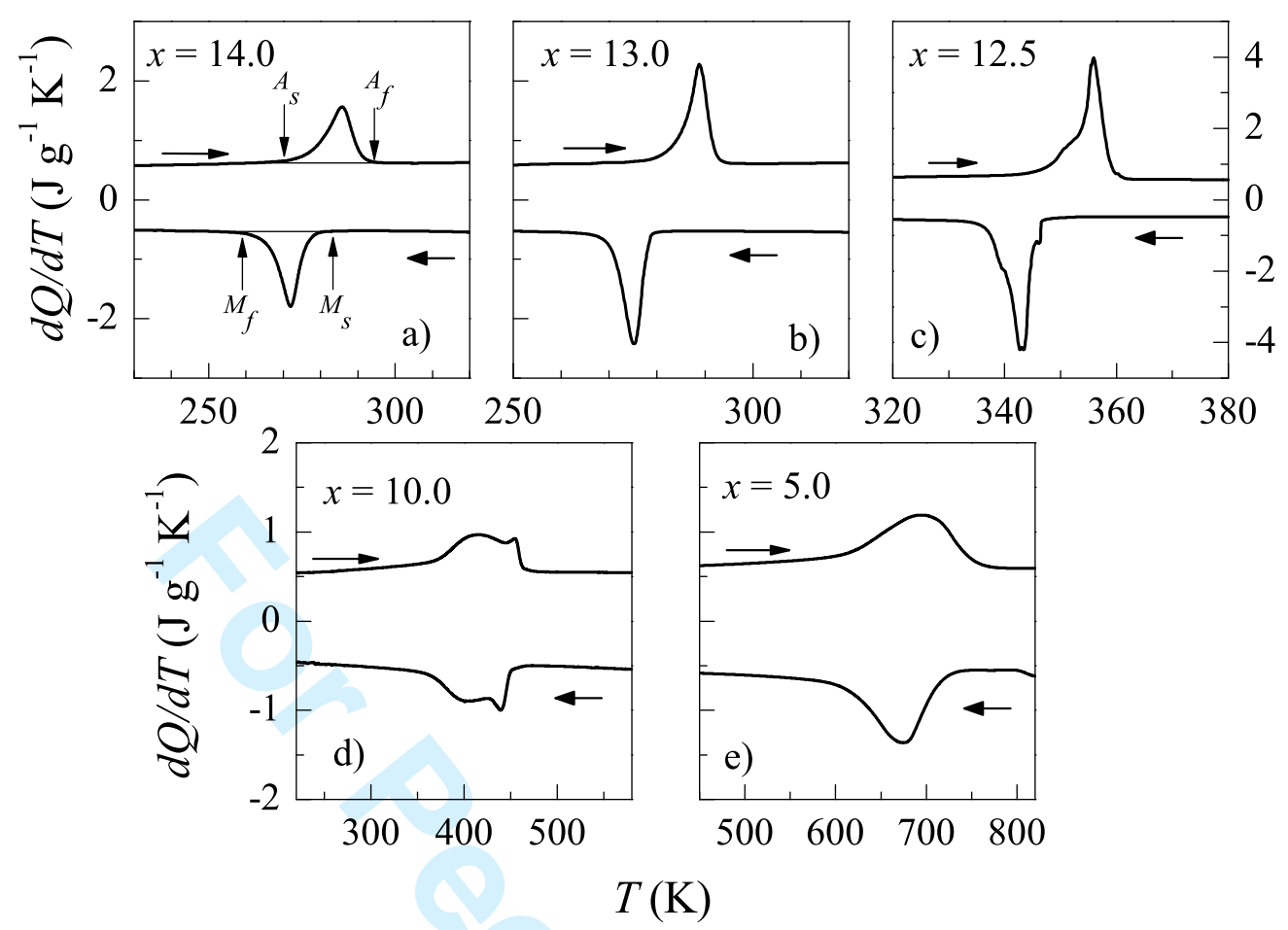

(a)

Figure 1. $d Q / d T$ versus temperature for the alloys undergoing martensitic transformations (a) $x=14.0$, (b) $x=13.0$, (c) $x=12.5$, (d) $x=10.0$, and (e) $x=5.0$. Horizontal arrows indicate direction of temperature change. Vertical arrows in part (a) show the positions of the martensitic and austenitic transition temperatures.

Table 2. Martensite start and finish temperatures $\left(M_{s}, M_{f}\right)$, austenite start and finish temperatures $\left(A_{s}, A_{f}\right)$ determined by DSC and $M(T)$ measurements ( $D S C$ and $M$ in superscript) and the enthalpy and entropy changes $(\Delta H, \Delta S)$ around the transition.

\begin{tabular}{lccccccccc}
\hline$x$ & $\begin{array}{c}M_{s}^{D S C} \\
(\mathrm{~K})\end{array}$ & $\begin{array}{c}M_{s}^{M} \\
(\mathrm{~K})\end{array}$ & $\begin{array}{c}M_{f}^{D S C} \\
(\mathrm{~K})\end{array}$ & $\begin{array}{c}A_{s}^{D S C} \\
(\mathrm{~K})\end{array}$ & $\begin{array}{c}A_{f}^{D S C} \\
(\mathrm{~K})\end{array}$ & $\begin{array}{c}A_{f}^{M} \\
(\mathrm{~K})\end{array}$ & $\begin{array}{c}\Delta T \\
(\mathrm{~K})\end{array}$ & $\begin{array}{c}\Delta H \\
\left(\mathrm{~J} \mathrm{~g}^{-1}\right)\end{array}$ & $\begin{array}{c}\Delta S \\
\left(\mathrm{~J} \mathrm{~g} \mathrm{~g}^{-1} \mathrm{~K}^{-1}\right)\end{array}$ \\
\hline 15.0 & - & $150-200$ & - & - & - & - & - & - & - \\
14.0 & 276 & 280 & 265 & 275 & 290 & 290 & 14 & $6.35 \pm 0.6$ & $0.022 \pm 0.002$ \\
13.0 & 277 & 284 & 270 & 280 & 291 & 295 & 14 & $7.04 \pm 0.7$ & $0.025 \pm 0.002$ \\
12.5 & 346 & 349 & 338 & 349 & 358 & 355 & 13 & $13.3 \pm 1.3$ & $0.038 \pm 0.003$ \\
10.0 & 444 & - & 368 & 380 & 457 & - & $11 / 14$ & $21.2 \pm 2$ & $0.051 \pm 0.005$ \\
5.0 & 709 & - & 621 & 632 & 741 & - & 19 & $42.2 \pm 4$ & $0.062 \pm 0.006$ \\
\hline
\end{tabular}

plied. The data were taken on increasing temperature up to $380 \mathrm{~K}$. Then, without removing the external field, the data were taken on decreasing-temperature (fieldcooled, FC) down to $5 \mathrm{~K}$, and finally on increasing temperature (field-heated, FH) up to $380 \mathrm{~K}$. To further characterise the magnetic properties, the magnetic field dependence of the magnetisation $M(H)$ was measured in fields up to $5 \mathrm{~T}$.

\section{Results}

\subsection{Calorimetric studies}

The results of the calorimetric studies for the alloys with $5.0 \leq x \leq 14.0$ which undergo martensitic transformations are plotted in Figs. 1(a)-1(e) in the temperature vicinity of the transformation. The heating and cooling cycles are indicated by 